\title{
EEG Recognition of Motor Imagery Based on EWT in Driving Assistance
}

\author{
Wei Shan ${ }^{1, a}$, Yuwen Wang ${ }^{1, b^{*}}$, Qun He ${ }^{1, c}$ and Ping Xie ${ }^{1, d}$ \\ ${ }^{1}$ College of Electrical Engineering, Yanshan University, Qinhuangdao 066004, PR China

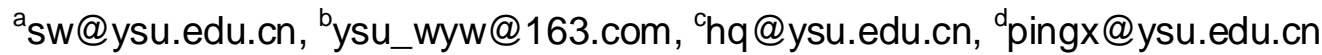 \\ * the corresponding author
}

\begin{abstract}
Keywords: Empirical wavelet transform; Motor imagery; Feature extraction; Pattern recognition; Driving assistance
\end{abstract}

\begin{abstract}
Electroencephalogram (EEG) feature extraction is one of the key techniques for Brain Computer Interface (BCI) in driving assistance. In this paper, empirical wavelet transform (EWT) is introduced for EEG feature extraction, and then a new EEG recognition method based on EWT is proposed. In the proposed method, the EEG features of the left arm flexion and extension motor imagery mode were first extracted via EWT and compared with those extracted by the empirical mode decomposition (EMD), and then the extracted features were classified by support vector machine (SVM). The results show that the EWT method significantly outperforms the EMD method, which can effectively decompose the intrinsic modes of EEG, and it presents several merits of fewer modes, no false mode and low computation cost. Therefore, the EWT method can effectively extract the local transient features of EEG and realize the effective classification through SVM, thus providing the chance of driving assistance through the arm flexion and extension imagination.
\end{abstract}

\section{Introduction}

Brain-computer interface[1] (BCI) represents a communication and control mechanism between the human brain and computers envisioned to support disabled people using their electrical activity of the brain, which is usually recorded using electroencephalogram (EEG). There are two types of BCI: spontaneous EEG, and evoked EEG based on BCI systems[2]. Today, BCI technology not only can be used as an important means of rehabilitation, but also can be used in the driving assistance area of the car, 1) the driver's fatigue state monitoring[3]; 2)an assistant control[4]. Therefore, BCI technology research has an important theoretical and practical significance.

The feature extraction of EEG signals is one of the key technologies of BCI, which directly determines the accuracy of control. For the non-linear and non-stationary characteristics of EEG, there are Short-time Fourier Transform[5], Wavelet Transform[6], Wavelet Packet Decomposition[7], EMD[9] methods and so on. Many nonlinear methods are widely used in the field of motor imagery feature extraction. Ensemble Empirical Mode decomposition (EEMD)[9] has good adaptability, while improving the model mixing of EMD. However, EEMD and EMD are lack of complete mathematical theories, decomposed modes are more, and each mode extraction requires multiple iterations, while EEMD also need to conduct multiple EMD and the amount of calculation is large.

In view of the shortcomings of EMD, Gilles[10] combined with EMD adaptive and wavelet analysis of the theoretical framework, proposed a new adaptive signal processing method, the empirical wavelet transform (EWT). In this paper, the EWT is introduced into the EEG feature extraction of motor imagery. An EEG feature extraction method based on EWT is proposed. Finally, the method is combined with SVM and successfully applied to classify the left arm flexion and extension motor imagery. The experimental results validate the effectiveness of the proposed method. 


\section{Empirical Wavelet Transform}

Similar to EMD, the purpose of EWT is to decompose the signal $f(t)$ as a sum of the $N+1$ intrinsic mode functions $f(t)$ :

$$
f(t)=\sum_{k=0}^{N} f_{k}(t)
$$

Where an IMF is defined as an amplitude modulated-frequency modulated function (AM-FM):

$$
f_{k}(t)=F_{k}(t) \cos \left(\varphi_{k}(t)\right) \quad \text { where }^{F_{k}(t)}, \varphi^{\prime}{ }_{k}(t)>0 \forall t
$$

The main assumption is that $F_{k}(t)$ and $\varphi_{k}^{\prime}(t)$ much slower than $\varphi_{k}$. Gilles ${ }^{[10]}$ proposed the method based on the theoretical framework of wavelet analysis. According to the Fourier spectral characteristics, the signal adaptively selects a group of wavelet filter banks to extract the signal of different AM-FM components. In order to select the appropriate wavelet filter banks, the Fourier spectrum needs to be segmented adaptively.

Assuming the Fourier support $[0, \pi]$ is segmented into $N$ contiguous segments, we denote $\omega_{n}$ to be the limits between each segments ( $\omega_{0}=0$ and $\omega_{N}=\pi$ ), as shown in Fig 1 .

Centered around each $\omega_{n}$, we define a transition phase (the gray hatched areas on Fig. 1) $T_{n}$ of width $2 \tau_{n}$.

After the interval $\Delta_{n}$ is defined, the empirical wavelet is defined as the bandpass filters on each $\Delta_{n}$. To do so, Gilles utilizes the idea used in the construction of both Littlewood-Paley and Meyer's wavelets $^{[8]}$. Then $\forall_{n}>0$, Gilles define the empirical scaling function $\hat{\phi}(w)$ and the empirical wavelets $^{[12]} \hat{\psi}_{n}(w)$.

The segmentation of the Fourier spectrum has a crucial effect on the adaptability of EWT, and the different portions of the spectrum which correspond to modes centered around a specific frequency and of compact support. In this paper, we use the method of determining the boundary $\omega_{n}$ in the literature ${ }^{[8]}$.

\section{Feature extraction and Classification Recognition}

Data description. 32-channel EEG acquisition device was used to collect the data of the sensory cortex from healthy people in this experiment. Before the acquisition of the signal, the subjects repeat the left upper limb flexion and extension motor imagery training, the subjects need to close eyes and relax body in the process of collecting the signal, according to. The EEG signals are collected from C3 and C4 channels, and they are divided into two categories. F (Flexion) and E (Extension) are used as markers. timing scheme of the corresponding instruction, the subjects begin the left upper limb flexion and extension motor imagery, the timing scheme is seen in Fig. 2.

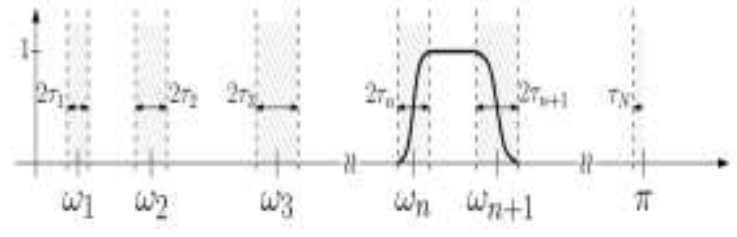

Figure 1. Partitioning of the Fourier axis.

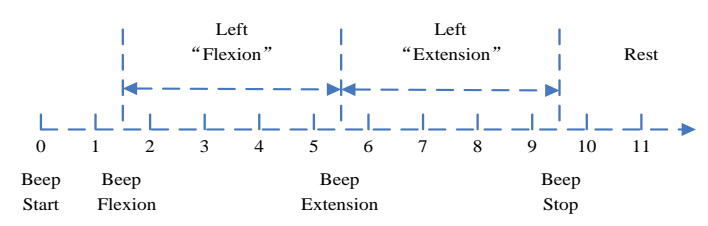

Figure 2. timing scheme.

The preprocessing of EEG signals. It is well known that the EEG signals are a small, low frequency of weak bioelectric signals, with $50 \mathrm{~Hz}$ frequency interference, baseline drift and other noise, in addition to ECG, EMG and other artifacts ${ }^{[9]}$. Studies have shown that motion-induced EEG is predominantly significant in $\mathrm{mu}(8 \sim 13 \mathrm{~Hz})$ and beta $(14 \sim 30 \mathrm{~Hz})$ rhythms ${ }^{[8]}$. To remove the above noise and artifacts interference. An adaptive high-pass filter is used to remove $0 \sim 0.3 \mathrm{~Hz}$ baseline drift, a notching filter is used to remove the power frequency interference, independent 
component analysis is used to remove EMG artifacts.

Feature extraction. Based on the non-linear and non-stationary characteristics of motor imagery in different action modes of unilateral body, this study designs a feature extraction method based on EWT, First of all, the preprocessed EEG signals are decomposed into multiple intrinsic mode functions through EWT, and the effective instantaneous components are selected and the frequency energy is calculated to realize the quantitative description of instantaneous features. The process of feature extraction is shown in Fig. 3.

First of all, decompose the preprocessed EEG signals through EWT, and then the decomposition of the sample component is relatively large, and a certain mode of action corresponding to the EEG feature embodied in the EEG signal specific band of energy changes, so selecting the most effective components of the concentrated energy is very necessary. In order to select effective components corresponding to the specific action modes, which are decomposed from EWT. According to the concept of time domain correlation coefficient proposed in [10], the frequency domain correlation coefficient index of the component is defined.

$$
\rho_{f, f_{k}}=\left|\frac{E\left[\left(f_{k}(t)-\mu_{f_{k}}\right)\left(f(t)-\mu_{f}\right)\right]}{\sigma_{f k} \sigma_{f}}\right| ; \rho_{f, f_{k}} \in[0,1]
$$

Where $f_{k}(t)$ and $f(t)$ represents the mode component decomposed using EWT and the original signal, respectively.

$\mu_{f k}, \sigma_{f k}, \mu_{f}, \sigma_{f}$ represents the corresponding time domain mean and standard deviation for $f_{k}(t)$ and $f(t)$, respectively; E represents the mathematical expectations. Which $\rho_{f, f_{k}}$ reflects the correlation between the component $f_{k}(t)$ and the original signal $f(t)$ in the time domain. The value is larger, the greater the correlation between the component and the original signal, that is to say, the higher the autocorrelation, the higher the proportion of the effective component. Therefore, the most relevant mode is chosen as the optimal mode of EWT and is used as the analysis sample of the next classification and identification. Finally, the band energy feature extraction is carried out according to Eq. 4 for the effective component.

$$
b_{i j}=\frac{1}{n} \sum_{k=1}^{n} v_{i j k}^{2}
$$

where $v_{i j k}$ represents EEG sampling point at the jth acquisition for lead of the ith experiment; $b_{i j}$ for the obtained band energy; $n$ is the number of EEG data collected here.

In order to reduce the impact of the subjects' thinking state when the EEG signals are collected at different times, the energy characteristics of the frequency band are normalized, and the concrete process is shown in Eq. 5.

$$
b_{i j}^{N}=\frac{b_{i j}-\mu_{j}}{\operatorname{std}\left(b_{j}\right)}
$$

Where $\mu_{j}$ is the average of the frequency band energy characteristics at the jth acquisition which leads for all experiments; std $\left(b_{j}\right)$ denotes the standard deviation of the frequency band energy characteristics for all experiments at the jth acquisition ${ }^{[11]}$.

\section{Recognition and classification based on SVM}

The important theoretical basis of SVM is the VC dimension theory of statistical learning and the principle of structural risk minimization. SVM can automatically find the support vector which better to distinguish the classification, and so that the classifier can maximize the interval among classes $^{[12]}$. Compared with the $\mathrm{BP}^{[13]}$ neural network algorithm, SVM can effectively avoid the risk of falling into the local optimum, and the real problem is transformed into the high-dimensional feature space by non-linear transformation, and the linear discriminant function is constructed in the high-dimensional space to replace the original space in the non-linear discriminant function, thus, not only can SVM have a better ability to promote, but also it can solve the problem of dimension. 
Assumed to be known training set $T=\left\{\left(x_{1}, y_{1}\right), \cdots,\left(x_{l}, y_{l}\right)\right\} \in(X \times Y)^{l}$, Where $x_{i}$ is the sample input vector, $y_{i}$ is the sample output vector, $x_{i} \in X=R^{n}, y_{i} \in Y=\{-1,1\}, i=1, \cdots, l$.

$$
\begin{aligned}
& \min J(\omega)=\frac{1}{2} \omega^{T} \omega \\
& \text { s.t }: y_{i}\left(\omega^{T} x_{i}+b\right) \geq 1, i=1,2, \cdots, n
\end{aligned}
$$

For the case of linear indivisibility, adding a relaxation variable $\xi_{i} \geq 0$ to the condition, the optimal problem becomes:

$$
\begin{aligned}
& \min J(\omega, \xi)=\frac{1}{2} \omega^{T} \omega+c \sum_{i=1}^{n} \xi_{i} \\
& \text { s.t. } y_{i}\left(\omega^{T} x_{i}+b\right) \geq 1-\xi_{i}, \xi \geq 0, i=1,2, \cdots, n
\end{aligned}
$$

the Gaussian radial basis function (RBF) was chosen as a kernel function whose expression is:

$$
k(x, z)=\exp \left(-g\|x-y\|^{2}\right)
$$

After the kernel function is selected, the SVM also needs to determine the penalty parameter c and the kernel parameter g, To a large extent, penalty parameters and local parameters determine the SVM learning ability and generalization ability.

In this paper, a cross-validation method is used for SVM parameters optimization to get the best penalty factor $\mathrm{c}$ and the kernel parameter $\mathrm{g}$, which can get better classification for test samples.

\section{Experiments}

In order to verify the effectiveness of the proposed method, nine healthy subjects were selected for experiments. Each subject was subjected to 21 groups' left upper limb flexion and extension motor imagery experiments according to the contents shown in Fig. 2, and subjects should flex for $3 \sim 5 \mathrm{~s}$ and extend for $7 \sim 9 \mathrm{~s}$, data would be recorded in each group, $256 \mathrm{~Hz}$ frequency was used to get the data of flexion and extension mode for analysis, which was divided into 100 pieces. The flexion data of the first 50 sections and the extension data of the first 50 sections was used as training samples, and the remaining data was used for the classification test.

Take the data of channel $\mathrm{C} 3$ in flexion mode as an example. After the EEG signal was decomposed by EWT and EMD, the frequency spectrum are shown in Fig. 4 and Fig. 5, respectively. We can find that the most effective components of the decomposed EEG are mainly concentrated in the two components $f_{2}$ and $f_{3}$ by calculating the correlation coefficient threshold.

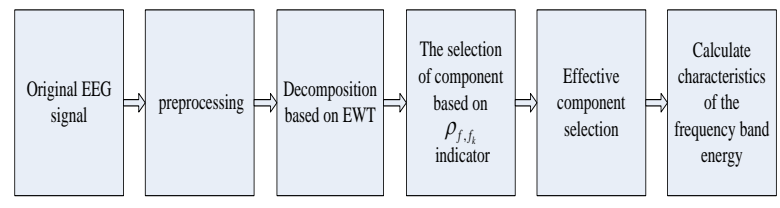

Figure 3. Feature extraction

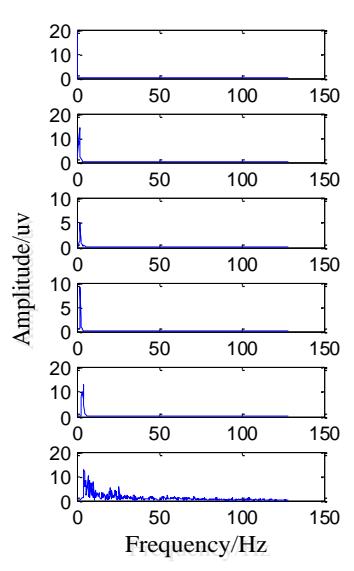

Figure 4. EWT

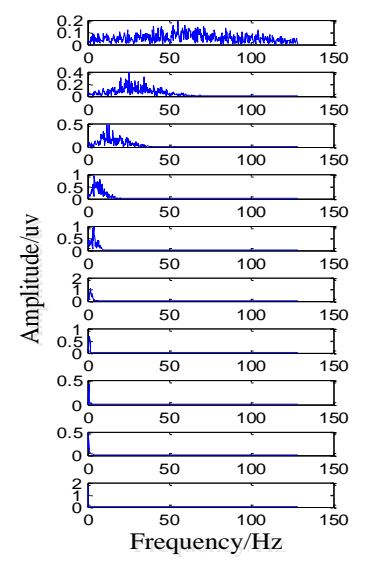

Figure 5. EMD

Where Fig. 4 from last to first row: $\left(f_{1}, f_{2}, f_{3}, f_{4}, f_{5}\right.$ and $f_{6}$, respectively). Fig. 5 from first to last row: $\left(i m f_{1}, i m f_{2}, i m f_{3}, i m f_{4}, i m f_{5}, i m f_{6}, i m f_{7}, i m f_{8}, i m f_{9}\right.$, respectively). 


\section{Conclusions}

In this paper, a new EEG feature extraction method based on EWT is proposed. And compared with the EMD, the decomposed components of the original EEG signals based on EWT are better than decomposed components based on EMD. The average correct rate of classification is $76.7361 \%$ and the best correct classification rate is $91.6667 \%$. The feasibility of the EWT to distinguish the motor imagery of unilateral limbs is verified, which provides a new solution for EEG feature extraction and classification. However, there are some problems about EWT, for example, how to achieve effective segmentation of the spectrum and other issues. This research focuses on the feature extraction and classification of EEG signals in motor imagery. The next step I will concentrate on improving the accuracy of classification, and studying the application of EEG signals in smart cars about assistance driving.

\section{Acknowledgements}

This work was supported by National Natural Science Foundation of China (Grant No.61673336).

\section{References}

[1] Kevric J, Subasi A: Comparison of signal decomposition methods in classification of EEG signals for motor-imagery BCI system[J]. Biomedical Signal Processing and Control, 2017 No.31, p.398.

[2] Qiu Z, Jin J, Lam H K, et al: Improved SFFS method for channel selection in motor imagery based BCI[J]. Neurocomputing, 2016 No.207, p.519.

[3] Hajinoroozi M, Mao Z, Jung T P, et al: EEG-based prediction of driver's cognitive performance by deep convolutional neural network[J]. Signal Processing: Image Communication, 2016 No.47, p.549.

[4] Santos V, Sappa A D, Oliveira M: Special Issue on Autonomous Driving and Driver Assistance Systems[J]. 2017 No.91, p.208.

[5] Zhong J, Huang Y: Time-frequency representation based on an adaptive short-time Fourier transform[J]. IEEE Transactions on Signal Processing, 2010 No.58(10), p.5118.

[6] Upadhyay D: Classification of eeg signals under different mental tasks using wavelet transform and neural network with one step secant algorithm[J]. International Journal of Scientific Engineering and Technology, 2013 No.2(4), p.256.

[7] Shiyu Y, Chong L, Haibin Z, et al: Imaginary EEG feature extraction based on wavelet packet decomposition $[\mathrm{J}][\mathrm{J}]$. Chinese Journal of Scientific Instrument, 2012 No.8, p.011.

[8] Huang N E, Shen Z, Long S R, et al: The Empirical Mode Decomposition and the Hilbert Spectrum for Nonlinear and No-Stationary Time Series Analysis[J]. Proceedings ofthe R oyal Society A, 1998 No.454, p.903.

[9] Wu Z H, Huang N E: Ensemble Empirical Mode De-composition-A Noise Assisted Data Analysis Method[J]. Advances in Adaptive Data Analysis, 2009 No.1(1), p.1.

[10] GILLES J: Empirical Wavelet Transform[J]. IEEE Transactions on Signal Processing, 2013 No.61(16), p.3999.

[11]Ping Xie, Xiao Ling Chen, Yu Ping Su,et al: Feature Extraction and Recognition of Motor Imagery EEG Based on EMD-Multiscale Entropy and Extreme Learning Machine[J]. Chinese Journal of Biomedical Engineering, 2013 No.06, p.641.(In Chinese)

[12] Alvarez-Meza AM, Velasquez-Martinez L F, Castellanos-Dominguez G: Time-Series Discrimination Using Feature Rel-evance Analysis in Motor Imagery Classification. Neurocom-puting, 2015 No.151(1), p.122.

[13] HuiXuan Fu,Hong Zhao, et all: Application Design of Neural Network- Mechanical Industry Press, China, 2010, p.83.(In Chinese) 